\title{
ON GOING DOWN FOR SIMPLE OVERRINGS
}

\author{
DAVID E. DOBBS ${ }^{1}$
}

\begin{abstract}
Let $R$ be an integral domain with quotient field $K$. If $R$ is Noetherian: then the Krull dimension of $R$ is at most $1 \Leftrightarrow$ for all overrings $S$ of $R, R \subset S$ satisfies going down. $R$ is Dedekind (resp., PID) $\Leftrightarrow R$ is Krull (resp., UFD) and, for all $u \in K, R \subset R[u]$ satisfies going down. $R$ is Prüfer $\Leftrightarrow R$ is integrally closed, every intersection of two principal ideals of $R$ is finitely generated, and $R \subset R[u]$ satisfies going down for all $u \in K$.
\end{abstract}

1. Introduction and notation. Let $R$ be a (commutative integral) domain with integral closure $\bar{R}$ and quotient field $K$. Our main purpose is to study going down (GD) between $R$ and its overrings (that is, $R$-subalgebras of $K)$. Using GD, we obtain characterizations of Prüfer domains in Corollary 4, of Noetherian domains of (Krull) dimension at most 1 in Proposition 7 and Corollary 9, and of Dedekind domains and PID's in Corollary 10. As may be expected from the characterization of Bézout domains given by Dawson and the author in [1, Corollary 4.4], a special role is played by simple overrings (that is, ones generated over $R$ or $\bar{R}$ by single elements of $K)$.

Any unexplained terminology is standard, as in [2], [3], and [7].

2. Domains characterized via going down. We begin by quoting the following result about FC domains (i.e., domains for which every intersection of two principal ideals is finitely generated). Observe that a domain is FC if and only if every element of its quotient field has finitely generated conductor.

Lemma 1 (McAdam [6, Theorem 2]). Let $R$ be FC and let $T$ be an overring of $R$ such that $R$ is integrally closed in $T$ and $R \subset T$ satisfies lying over (LO). Then $R=T$.

Lemma 2. Let $R$ be quasi-local. Assume either

(a) $R$ is a Krull domain such that $\operatorname{dim}(R) \geqq 2$ or

(b) $R$ is an integrally closed FC domain which is not a valuation ring.

Received by the editors July 10, 1972 and, in revised form, October 30, 1972.

AMS (MOS) subject classifications (1970). Primary 13F05.

Key words and phrases. Going down, Krull domain, FC domain.

${ }^{1}$ This work was supported in part by NSF Grant GP-28409.

(C) American Mathematical Society 1973 
Then there exists $u \in K$ such that $u \notin R, u^{-1} \notin R$ and $R \subset R[u]$ does not satisfy $G D$.

Proof. If $R$ is a Krull domain, the assumption about its dimension readily implies that $R$ is not a valuation ring (cf. [2, Theorem 35.16]). Hence, in case either (a) or (b) holds, there exists $u \in K$ such that $u \notin R$ and $u^{-1} \notin R$. If $M$ is the maximal ideal of $R$, then Chevalley's lemma [3, Theorem 55] implies that $M$ survives in either $R[u]$ or $R\left[u^{-1}\right]$. Without loss of generality, $M R[u] \neq R[u]$, and so there exists a prime of $R[u]$ lying over $M$.

Now, suppose that the lemma is false. Then $R \subset R[u]$ satisfies GD and, since $R$ is quasi-local, $R \subset R[u]$ also satisfies LO. In case (b), Lemma 1 shows $u \in R$, a contradiction. For case (a), let $P$ be any prime of $R$ of height 1 . As $R_{P}$ is a discrete (rank 1) valuation ring and $R_{P} \subset R_{P}[u]=$ $R[u]_{R \backslash P}$ inherits LO from $R \subset R[u]$, Lemma 1 implies $u \in R_{P}$. Hence $u \in \bigcap R_{P}=R$, a contradiction, to complete the proof.

THEOREM 3. Assume either (a) $R$ is a Krull domain such that $\operatorname{dim}(R) \geqq 2$ or (b) $R$ is an integrally closed FC domain which is not Prüfer. Then there exists $u \in K$ such that $R \subset R[u]$ does not satisfy $G D$.

Proof. (a) Let $M$ be a maximal ideal of $R$ of height at least 2. Note $R_{M}$ is Krull [2, Corollary 35.6], quasi-local, of dimension at least 2. If the result is false, then $R_{M} \subset R_{M}[u]=R[u]_{R \backslash M}$ inherits GD from $R \subset R[u]$, for all $u \in K$. This contradicts case (a) of Lemma 2 .

(b) Since $R$ is not Prüfer, there exists a maximal ideal $M$ of $R$ such that $R_{M}$ is not a valuation ring. As $R_{M}$ is integrally closed and FC, the proof concludes as above, this time using case (b) of Lemma 2.

Corollary 4. $R$ is Prüfer if and only if the following three conditions hold: (i) $R$ is integrally closed; (ii) $R$ is $F C$; (iii) $R \subset R[u]$ satisfies $G D$ for all $u \in K$.

Proof. Let $R$ be Prüfer. It is well known that (i) and (ii) hold. As explained prior to [1, Proposition 3.6], (iii) also holds.

The converse follows immediately from case (b) of Theorem 3.

We remark that Corollary 4 extends a result of Quentel [8, Corollaire 2]. A different extension appears in [6, Theorem 1].

Lemma 5 (resp., Proposition 8) will, in special cases, relate the question of unibranchedness of $R$ in $\bar{R}$ to that of GD for simple overrings generated by elements of $\bar{R}$ (resp., of $K \backslash \bar{R}$ ). Lemma 5 was essentially proved by McAdam in [5, Theorem 2].

LemMA 5. Assume $R$ is Noetherian, $T$ is an overring of $R$, and $R \subset R[u]$ satisfies $G D$ for all $u \in T$. If $P$ is a prime of $R$ of height at least 2 , then at most one prime of $T$ lies over $P$. 
COROllary 6. Let $R$ be Noetherian and $T$ an overring of $R$ contained in $\bar{R}$. The following conditions are equivalent:

(i) $R \subset T$ satisfies $G D$;

(ii) $R \subset S$ satisfies $G D$ for all rings $R \subset S \subset T$;

(iii) $R \subset R[u]$ satisfies $G D$ for all $u \in T$.

Proof. For rings $R \subset S \subset T$, integrality shows $S \subset T$ satisfies LO, whence [5, Lemma 1(2)] yields (i) $\Rightarrow$ (ii). Clearly, (ii) $\Rightarrow$ (iii). Finally, assume (iii). If $R \subset T$ fails to satisfy GD, there exist primes $P_{1} \subsetneq P_{2}$ of $R$ and $Q$ of $T$ such that $Q \cap R=P_{2}$ and no prime of $T$ contained in $Q$ lies over $P_{1}$. Since $R \subset T$ satisfies GU and LO [3, Theorem 44] and $P_{2}$ is unibranched in $T$ (by Lemma 5 ), we easily obtain a contradiction. Thus (iii) $\Rightarrow$ (i), to complete the proof.

Proposition 7. Let $R$ be Noetherian. The following conditions are equivalent:

(i) $\operatorname{dim}(R) \leqq 1$;

(ii) $S \subset T$ satisfies $G D$ for all overrings $S \subset T$ of $R$;

(iii) $R \subset S$ satisfies $G D$ for all overrings $S$ of $R$.

Proof. (i) $\Rightarrow$ (ii) follows immediately from the Krull-Akizuki theorem [3, Theorem 93], and (ii) $\Rightarrow$ (iii) is clear. Finally, if $P$ is a prime of $R$ of height greater than 1 , then $[4, \S 13]$ supplies a discrete (rank one) valuation overring $V$ of $R$ such that the maximal ideal of $V$ lies over $P$. Since $R \subset V$ does not satisfy GD, we conclude that (iii) $\Rightarrow$ (i).

Proposition 8. Assume $R \subset \bar{R}[u]$ satisfies $G D$ for all $u \in K \backslash \bar{R}$. Assume either (a) $\bar{R}$ is a Krull domain such that $\operatorname{dim}(R) \geqq 2$ or (b) $\bar{R}$ is $F C$ and not Prüfer. Then there exists a nonmaximal prime of $R$ which is not unibranched in $\bar{R}$.

Proof. Note that $\operatorname{dim}(\bar{R})=\operatorname{dim}(R)$. Hence, by applying the appropriate case of Theorem 3 , there exists $u \in K$ such that $\bar{R} \subset \bar{R}[u]$ does not satisfy GD. Thus, there exist primes $M_{1} \subsetneq M_{2}$ of $\bar{R}$ and $Q$ of $\bar{R}[u]$ such that $Q \cap \bar{R}=M_{2}$ and no prime of $\bar{R}[u]$ contained in $Q$ lies over $M_{1}$. Let $P_{i}=$ $M_{i} \cap R(i=1,2)$. Since $R \subset \bar{R}[u]$ satisfies GD, there exists a prime $N$ of $\bar{R}[u]$ such that $N \subset Q$ and $N \cap R=P_{1}$. Then $P_{1}$ is not unibranched in $\bar{R}$, as $N \cap \bar{R}$ and $M_{1}$ are distinct. Of course, the INC property for integral extensions [3, Theorem 44] shows $P_{1} \neq P_{2}$, and so $P_{1}$ is nonmaximal, to complete the proof.

We next show that, under an assumption of unibranchedness, we may restrict consideration in part (iii) of Proposition 7 to simple overrings $S$.

COROLlaRY 9. Let $R$ be Noetherian such that every prime of $R$ of height 1 is unibranched in $\bar{R}$. Then $\operatorname{dim}(R) \leqq 1$ if and only if $R \subset \bar{R}[u]$ satisfies $G D$ for all $u \in K \backslash \bar{R}$. 
ProOF. The "only if" assertion is trivial. If the "if" assertion fails, a consequence of the principal ideal theorem [3, Theorem 152] allows us to choose a prime $M$ of $R$ of height 2. As Mori's theorem [7, Theorem 33.10] shows $\bar{R}$ is Krull, it follows that $A=(\bar{R})_{R \backslash M}$, the integral closure of $R_{M}$, is also Krull. If $u \in K \backslash A$, then $R_{M} \subset A[u]$ inherits GD from $R \subset \bar{R}$ [u]. Thus, case (a) of Proposition 8 provides a nonmaximal prime $P$ of $R_{M}$ which is not unibranched in $A$. Then $P \cap R$ is a prime of $R$ of height 1 which is not unibranched in $\bar{R}$, a contradiction, to complete the proof.

Corollary 10. Let $R$ be Krull (resp., UFD). Then $R$ is Dedekind (resp., PID) if and only if $R \subset R[u]$ satisfies $G D$ for all $u \in K$.

Proof. As explained prior to [1, Proposition 3.6], the "only if" assertions are immediate. To obtain the converses, apply [2, Propositions 31.6 and 35.2 and Theorem 35.16] and case (a) of Theorem 3.

REMARKS. (i) The use of Theorem 3 in the preceding proof characterizing PID's may be replaced by an appeal to [1, Corollary 4.4], since any UFD is a GCD.

(ii) Since Lemma 1 appears in $[7,(33.1)$, p. 114] for the case of Noetherian $R$, the proofs of Lemma 2(a), Theorem 3(a), Propositions 7 and 8(a), and Corollaries 9 and 10 do not depend on the results in [6].

(iii) The question of characterizing the domains satisfying GD with simple overrings is far from settled. We close by sketching an example ${ }^{2}$ of a quasi-local integrally closed two-dimensional domain $R$ such that $R \subset R[u]$ satisfies GD for all $u \in K$ and $R$ is not valuation.

First, let $S$ be a quasi-local integrally closed one-dimensional domain which is not valuation (for example, construct $S$ as in [3, 2-1, Exercise 5]). Let $M$ be the maximal ideal of $S$ and $F$ the quotient field of $S$. Define $R$ to be the restricted power series ring $S+x F[[x]]$. Then $R$ is not valuation, is integrally closed in its quotient field $K=F((x))$, and has only two nonzero primes, viz., $M+x F[[x]]$ and $P=x F[[x]]$.

It remains to show $R \subset R[u]$ satisfies GD whenever $u \in K$. One reduces quickly to the case $u \notin R, u^{-1} \notin R$. If $u \notin F[[x]]$, then $u^{-1} \in P$, a contradiction. Hence $u=v+w$ for some $v \in F \backslash S$ and $w \in P$. Since $R[u]=R[v]=$ $S[v]+P$, we see that $P$ is also a prime of $R[u]$, whence $R \subset R[u]$ satisfies GD.

\section{REFERENCES}

1. J. Dawson and D. E. Dobbs, On going down in polynomial rings, Canad. J. Math. (to appear).

2. R. W. Gilmer, Multiplicative ideal theory, Queen's Papers in Pure and Appl. Math., no. 12, Queen's University, Kingston, Ont., 1968. MR 37 \#5198.

${ }^{2} \mathrm{I}$ am indebted to the referee for this example and for improving an earlier version of Proposition 7. 
3. I. Kaplansky, Commutative rings, Allyn and Bacon, Boston, Mass., 1970. MR 40 \#7234.

4. - Topics in commutative rings. II, University of Chicago, Chicago, Ill. (mimeographed notes).

5. S. McAdam, Going down, Duke Math. J. 39 (1972), 633-636.

6. —, Two conductor theorems, J. Algebra 23 (1972), 239-240.

7. M. Nagata, Local rings, Interscience Tracts in Pure and Appl. Math., no. 13, Interscience, New York, 1962. MR 27 \#5790.

8. Y. Quentel, Sur une caractérisation des anneaux de valuation de hauteur $1, \mathrm{C} . \mathrm{R}$. Acad. Sci. Paris Sér. A-B 265 (1967), A659-A661. MR 36 \#3779.

Department of Mathematics, Rutgers University, The State University of New Jersey, New Brunswick, New Jersey 08903 